\title{
Surface conductance and microwave scattering in semicontinuous gold films
}

\author{
Jan Obrzut \\ Material Measurement Laboratory, National Institute of Standards and Technology, Gaithersburg, MD 20899-8542, USA
}

\begin{abstract}
Semicontinuous gold films $4 \mathrm{~nm}$ to $12 \mathrm{~nm}$ thick were characterized using patterned coplanar waveguides over a frequency range of $100 \mathrm{MHz}$ to $20 \mathrm{GHz}$. Such films can form two dimensional fractal aggregates mixed with dielectric voids with unusually large electromagnetic absorption. Surface conductance and microwave absorption were obtained from the measured scattering parameters using a microwave transmission-reflection model. Within the percolation coverage of gold nanoparticles, when the surface conductance increases from $10^{-5} \mathrm{~S}$ to $10^{-3} \mathrm{~S}$, the film properties transition from dielectric to metallic, and the corresponding microwave transmittance falls far more rapidly than the classical skin depth model would suggest. The resulting microwave absorption attains a peak value in this range, which results from an inhomogeneous localization of an electromagnetic field in fractal structures. The dielectric to metallic transition can be easily identified experimentally from an abrupt change in the phase of the reflection scattering parameter $\mathrm{S}_{11}$. The results demonstrate a convenient measurement technique to study electromagnetic properties of surface-enhanced semicontinuous metallic films for thin broadband absorbers with minimized reflection, and for other microwave applications.
\end{abstract}

\section{Section: RESEARCH PAPER}

Keywords: coplanar waveguides; scattering parameters; conductance; microwave absorption; percolation; semi-continuous gold films

Citation: Jan Obrzut, Surface conductance and microwave scattering in semicontinuous gold films, Acta IMEKO, vol. 4, no. 3, article 7, September 2015, identifier: IMEKO-ACTA-04 (2015)-03-07

Editor: Paolo Carbone, University of Perugia, Italy

Received February 24, 2015; In final form April 8, 2015; Published September 2015

Copyright: @ 2015 IMEKO. This is an open-access article distributed under the terms of the Creative Commons Attribution 3.0 License, which permits unrestricted use, distribution, and reproduction in any medium, provided the original author and source are credited

Funding: This work was supported by the NIST Center for Nanoscale Science and Technology, and Material Measurement Laboratory

Corresponding author: Jan Obrzut, e-mail: jan.obrzut@nist.gov

\section{INTRODUCTION}

Semicontinuous thin metallic films have attracted attention due to their unusual optical and electronic properties such as enhancement of non-linear optical effects [1] and inhomogeneous localization of electromagnetic eigenmodes [2]. The characteristic fractal morphology of such films develops during deposition, when metallic nanoparticles nucleate to form conducting clusters mixed with dielectric voids. The size of the conducting clusters increases with increasing deposition thickness until a percolation threshold is passed and the film becomes conducting [3], [4]. In the vicinity of the percolation transition, the size of the inhomogeneities is comparable with the mean free path of the electrons and results in partial carrier localization [5]. Such films may also exhibit unexpected electromagnetic characteristics, as the carrier response can be dominated by resistive localized activated transport between grains [2]. Typically, DC or THz [6] electrical conductivity has been reported, but microwave studies are scarce due to the complexity of such measurements [7], [8]. In this paper, we report measurements of conductance and power absorption over the dielectric to metallic percolation transition using a technique based on coplanar waveguides (CPW) [9]. This approach enables effective impedance matching and allows for measurement of the film propagation characteristics, which can be accurately normalized with the film surface conductance while mitigating uncertainty from the film thickness measurement. The results are presented for gold nanoparticles deposited on a flat surface, which we choose as a model of $2 \mathrm{D}$ semicontinuous metallic film. 


\section{METHODS}

\subsection{Coplanar Waveguide Testing Structure}

Coplanar waveguides with a nominal impedance value $\left(Z_{0}\right)$ of $50 \Omega$ and a propagation length ( $)$ ranging from $450 \mu \mathrm{m}$ to $1800 \mu \mathrm{m}$, were made with $10 \mathrm{~nm}$ Ti and $200 \mathrm{~nm}$ Au evaporated on $500 \mu \mathrm{m}$ thick, $25 \mathrm{~mm}$ by $25 \mathrm{~mm}$ electronic grade alumina wafers (Figure 1). CPWs were patterned by lift off lithography. The width $(w)$ of the central signal strip of these CPWs was 50 $\mu \mathrm{m} \pm 0.2 \mu \mathrm{m}$, while the signal to ground plane spacing $(s)$ was nominally $22 \mu \mathrm{m}$.

Semicontinuous films were deposited by thermal evaporation of gold directly onto CPW through a shadow mask. During deposition the film average mass thickness (d) was monitored by a quartz crystal oscillator with resolution of \pm 1 $\mathrm{nm}$.

\subsection{Microwave Measurement and Analysis}

Measurements of the microwave wave scattering parameters, $\boldsymbol{S}_{11}$ and $\boldsymbol{S}_{21}$, were performed using an Agilent $8720 \mathrm{D}$ network analyzer in the frequency range of $1 \mathrm{GHz}$ to 20 $\mathrm{GHz}$. The analyzer was connected to the CPW test structure with phase preserving cables from Agilent (85131-60013) and $50 \Omega$ ground-signal-ground (GSG) air-coplanar probes (ACP$40,100 \mu \mathrm{m}$ pitch) from Cascade. The measurement system was calibrated using a 101-190C impedance calibration standard and WinCal calibration software from Cascade.

Parameters in bold face denote complex quantities that have both magnitude and phase. After film deposition, the CPW impedance changes from $Z_{0}$ to $\mathbf{Z}_{\mathbf{s}}$. We consider the CPW test structure as a microwave network consisting of impedance discontinuity $Z_{0} ; Z_{\mathrm{s}} ; Z_{0}$, that is, $Z_{\mathrm{s}}$ inserted between two reference transmission lines having a real characteristic impedance $Z_{0}$ (Figure 1) where multiple wave reflection takes place at each $Z_{0} ; Z_{\mathrm{s}}$ interface, affecting the reflection coefficient, $\boldsymbol{\Gamma}$, and transmission coefficient, $\boldsymbol{T}$. The material's properties in the specimen section of propagation length $l$ are represented by the complex impedance $\boldsymbol{Z}_{\mathrm{s}}$, and complex propagation constant $\boldsymbol{\gamma}_{\mathrm{s}}$. The relation between the measured scattering parameters $\boldsymbol{S}_{11}$, $\boldsymbol{S}_{21}$, and $\boldsymbol{\Gamma}, \boldsymbol{Z}_{\mathrm{s}}$ and $\boldsymbol{T}$ are given by equations (1-3) [10]-[12]:

$\boldsymbol{\Gamma}=\boldsymbol{b}-\sqrt{\boldsymbol{b}^{2}-1}, \boldsymbol{b}=\frac{\boldsymbol{S}_{11}{ }^{2}-\boldsymbol{S}_{21}{ }^{2}+1}{2 \boldsymbol{S}_{11}}$

$Z_{S}=Z_{0} \frac{1+\Gamma}{1-\Gamma}$

$\boldsymbol{T}=\frac{\boldsymbol{S}_{11}+\boldsymbol{S}_{21}-\boldsymbol{\Gamma}}{1-\left(\boldsymbol{S}_{11}+\boldsymbol{S}_{21}\right) \boldsymbol{\Gamma}}$

In (1) $|\boldsymbol{\Gamma}|<=1.0$. The complex transmission coefficient $\boldsymbol{T}$ $=\mathrm{e}^{\gamma_{\mathrm{s}} l}$ is related to the complex propagation constant by (4):

$$
\gamma_{s}=\left[\ln \left(T^{-1}\right)\right] / l
$$



Figure 1. CPW Testing structure. which has multiple solutions. Since $\boldsymbol{T}=|\boldsymbol{T}| \mathrm{e}^{\mathrm{j} \boldsymbol{\phi}}$, (4) can be rearranged into (5) where the real part of $\gamma_{\mathrm{s}}$ has an unique value, and only the imaginary part, the phase constant in (3) has multiple values:

$\boldsymbol{\gamma}_{\mathrm{s}}=\left[\ln \left|\boldsymbol{T}^{-1}\right|\right] / l+j[(2 \pi \mathrm{n}-\phi) / l]$

The ambiguity in the phase constant can be easily eliminated by measurements on two CPWs with different propagation lengths. Having determined $\boldsymbol{Z}_{\mathrm{s}}$ and $\boldsymbol{\gamma}_{\mathrm{s}}$, the distributed conductance, $G_{\mathrm{s}}$, can be obtained from the conventional transmission line relations [12]:

$G_{s}=\operatorname{Re}\left(\frac{\boldsymbol{\gamma}_{\mathrm{s}}}{\boldsymbol{Z}_{\mathrm{s}}}\right)$.

The distributed conductance $G_{\mathrm{s}}(6)$ can be scaled with the film surface conductance $\left(\sigma_{\mathrm{s}}\right)$, (in units of Siemens per square):

$\sigma_{s}=\frac{1}{2} G_{s} s$

where $(s)$ is the CPW signal to ground plane spacing, here $s=22 \mu \mathrm{m}$ (Figure 1). Note, that $\sigma_{\mathrm{s}}$ is independent of the CPW geometry, but it reflects the physical structure of the film, which depends on the particles surface coverage. In particular, $\sigma_{\mathrm{s}}$ eliminates ambiguity associated with the film mass thickness measurement, which is commonly used in characterizing 2D metallic films. The microwave absorption, $A_{s}$, is given by (8):

$A_{\mathrm{s}}=1-T_{\mathrm{s}}-R_{\mathrm{s}}$

where the transmittance $T_{\mathrm{s}}$, and reflectance $R_{\mathrm{s}}$, are magnitudes of the corresponding transmission and reflection coefficient, respectively.

The combined uncertainty of scattering parameters magnitude is $0.1 \mathrm{~dB}$ and phase angle is $2^{\circ}$. The combined relative uncertainty of $\sigma_{\mathrm{s}}$ and $A_{\mathrm{s}}$ is within $5 \%$.

\section{RESULTS}

\subsection{Scattering parameters}

Figure 2 shows the magnitude and phase of the complex scattering parameters measured for uncoated CPWs and CPWs
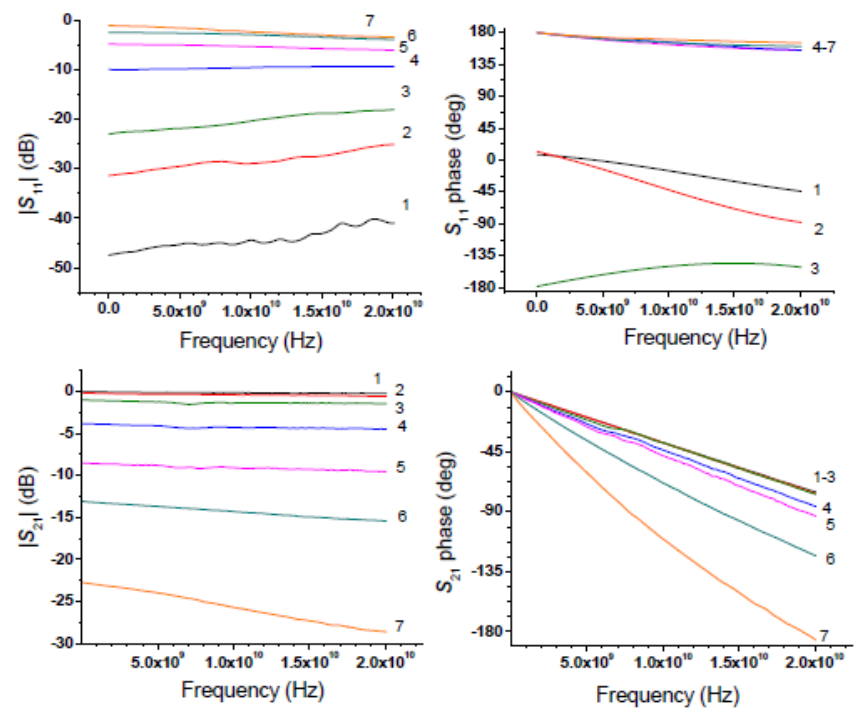

Figure 2. Magnitude and phase of scattering parameters $\boldsymbol{S}_{11}$ and $\boldsymbol{S}_{21}$ for the uncoated CPWs (1), and the following Au film thickness: (2) $4 \mathrm{~nm},(3) 6 \mathrm{~nm}$, (4) $7 \mathrm{~nm}$, (5) $8 \mathrm{~nm},(6) 9 \mathrm{~nm}$, and (7) $10 \mathrm{~nm}$. 
coated with gold films with thickness increasing from $4 \mathrm{~nm}$ to $10 \mathrm{~nm}$. The magnitude of the reflected wave, $\left|\boldsymbol{S}_{11}\right|$, for uncoated CPWs is rather small, in the range of $-45 \mathrm{~dB}$, indicating negligibly small insertion loss. The corresponding phase angle (plot 1 of $\boldsymbol{S}_{11}$ phase) indicates that the impedance mismatch is not significant. Uncoated CPWs show highly transmitting characteristics with $\left|\boldsymbol{S}_{21}\right|$ slightly decreasing with frequency from its maximum value of $0 \mathrm{~dB}$, again indicating that the CPWs test structures are well impedance matched.

There is a general tendency of conducting Au films to affect the magnitude and phase of both the $\boldsymbol{S}_{11}$ and $\boldsymbol{S}_{21}$ parameters. With increasing film thickness, the magnitude of $\boldsymbol{S}_{11}$ increases, while the magnitude of $\boldsymbol{S}_{21}$ correspondingly decreases. For example, in comparison to uncoated CPWs, for a $7 \mathrm{~nm}$ thick Au film the $\left|\boldsymbol{S}_{11}\right|$ value increases by several orders of magnitude, from $-45 \mathrm{~dB}$ to about $-10 \mathrm{~dB}$, while $\left|\boldsymbol{S}_{21}\right|$ decreases from about $0 \mathrm{~dB}$ to about $-4.3 \mathrm{~dB}$. These changes are significant, and reflect the increasingly conducting character of the Au films. As the film thickness grows, $\left|\boldsymbol{S}_{11}\right|$ increases towards the maximum value of $0 \mathrm{~dB}$, while the phase angle approaches the value of $\pi$. An abrupt change in $\boldsymbol{S}_{11}$ phase, from $-135^{\circ}$ to $+175^{\circ}$ when the film thickness increases from $6 \mathrm{~nm}$ to $8 \mathrm{~nm}$, indicates a conductivity percolation transition from a dielectric to a conducting metallic state. Films thicker than $6 \mathrm{~nm}$ are clearly conducting, each showing $\left|\boldsymbol{S}_{11}\right|$ values approaching $0 \mathrm{~dB}$ and similar phase angles of about $\pi$. This is characteristic for reflection from a highly conducting interface. Changes in $\boldsymbol{S}_{21}$ magnitude and phase show higher sensitivity to these thicker, more conducting films, and our measurement of both $\boldsymbol{S}_{11}$ and $\boldsymbol{S}_{21}$ accurately captures this transition from the insulating to the conducting state. The conductance is apparently frequency independent, as inferred from the flat characteristic of the magnitude of scattering parameters. It also increases with increasing film thickness.

\subsection{Surface conductance}

The surface conductance values, $\sigma_{\mathrm{s}}$, determined from (7) are listed in Table 1 at several frequencies. The observed slight dependence of $\sigma_{\mathrm{s}}$ on frequency is rather not significant, and for films thicker than $4 \mathrm{~nm}$ it is within the measurement uncertainty of $5 \%$. In comparison the effect of the particle coverage, indicated by the film mass thickness $d$, is much larger.

Films $4 \mathrm{~nm}$ thick are only weakly conducting with $\sigma_{\mathrm{s}}$ slightly increasing with frequency, from $2 \times 10^{-6} \mathrm{~S}$ at $2 \mathrm{GHz}$ to about 4.0 $\times 10^{-6} \mathrm{~S}$ at $10 \mathrm{GHz}$. Thicker films show higher $\sigma_{\mathrm{s}}$ values. $\sigma_{\mathrm{s}}$ of $6 \mathrm{~nm}$ thick films is in the range of about $1.3 \times 10^{-5} \mathrm{~S}$ to $1.4 \times$ $10^{-5} \mathrm{~S}$. This value increases further by three orders of magnitude to about $1.5 \times 10^{-2} \mathrm{~S}$ for films $10 \mathrm{~nm}$ thick. Such a large increase of conductance within the relatively narrow

Table 1. Surface conductance (in S), at several frequencies $(f)$ for gold films having mass thickness $(d)$.

\begin{tabular}{|c|c|c|c|c|c|}
\hline $\boldsymbol{d}(\mathbf{n m})$ & \multicolumn{5}{|c|}{$\boldsymbol{f}(\mathbf{G H z})$} \\
\hline & $\mathbf{2}$ & $\mathbf{5}$ & $\mathbf{1 0}$ & $\mathbf{1 5}$ & $\mathbf{2 0}$ \\
\hline $\mathbf{4}$ & $1.8610^{-6}$ & $2.8510^{-6}$ & $3.9210^{-6}$ & $3.7110^{-6}$ & $3.3210^{-6}$ \\
\hline $\mathbf{6}$ & $1.3310^{-5}$ & $1.4710^{-5}$ & $1.4610^{-5}$ & $1.3310^{-5}$ & $1.2710^{-5}$ \\
\hline $\mathbf{7}$ & $1.0010^{-3}$ & $1.0110^{-3}$ & $1.1010^{-3}$ & $1.1010^{-3}$ & $1.1610^{-3}$ \\
\hline $\mathbf{8}$ & $3.0010^{-3}$ & $3.0010^{-3}$ & $3.0110^{-3}$ & $3.0110^{-3}$ & $3.1410^{-3}$ \\
\hline $\mathbf{9}$ & $6.4810^{-3}$ & $6.5910^{-3}$ & $6.6810^{-3}$ & $6.7210^{-3}$ & $6.6810^{-3}$ \\
\hline $\mathbf{1 0}$ & $1.8710^{-2}$ & $1.7310^{-2}$ & $1.5510^{-2}$ & $1.4410^{-2}$ & $1.3510^{-2}$ \\
\hline
\end{tabular}

thickness range is indicative of the dielectric to metallic conductor percolation transition. With increasing $d$ the metallic nanoparticles, initially separated in a mix with dielectric voids, nucleate to form conducting clusters. The size of the conducting clusters increases with increasing film thickness until a percolation threshold is passed and the predominantly dielectric film becomes a metallic conductor. In our previous work [13], we applied the generalized effective medium theory (GEM) to model the effect of film surface coverage, $p$, on conductivity and found this model to provide a good estimate of the percolation threshold concentration $p_{c}$, and critical exponents, $s$ and $t$. In the GEM model, the conductivity, $\sigma_{\mathrm{s}}$, below $p_{\mathrm{c}}$ scales with the surface conductance of the dielectric, $\sigma_{\mathrm{d}}$, as $\sigma_{\mathrm{s}} \approx \sigma_{\mathrm{d}}\left(p_{\mathrm{c}}-p\right)^{s}$ where the exponent $s$ describes the singular changes in $\sigma_{\mathrm{s}}$ near the percolation threshold. $\sigma_{\mathrm{d}}=210^{-7} \mathrm{~S}$ is the measured conductance of the alumina substrate. Above $p_{\mathrm{c}}, \sigma_{\mathrm{s}}$ scales with the exponent $t$, $\sigma_{\mathrm{s}} \approx \sigma_{\mathrm{m}}\left(p-p_{\mathrm{c}}\right)^{t}$, where $\sigma_{\mathrm{m}}$ is the surface conductance of pure metal. The universal exponents $t$ and $s$, both have values of about $1.2 \pm 0.2$, close to the known 'universal' percolation exponent values in two dimensions [14]. Similarly, the $p_{\mathrm{c}}$ value of about 0.54 is consistent with the value of 0.5 predicted for a 2D square lattice. Thus overall, these results were found to be consistent with charge transport in two-dimensions. In comparison, $s \approx 0.73$ in three-dimensions for thicker, $20 \mathrm{~nm}$ films of random metallic particles [15]. The GEM model formally "merges" the percolation and effective medium theories and typically captures well the conductivity crossover between the high and low conductivity states that is characteristic of most real dielectric - metallic composite materials in two and three dimensions [16], [17]. The shortcoming of this procedure is that the exact perturbative result is no longer recovered in the additive concentration. The effective medium theory predicts 'effective' absorption near $p_{\mathrm{c}}$ in a very narrow concentration range when the permittivity diverges from the dielectric to metallic, even if both the dielectric and conducting components are lossless. In the next section we show the experimental evidence of a broad microwave absorption peak that is continuous on the conductivity scale, in apparent contradiction with the effective medium scaling arguments.

\subsection{Microwave absorption}

Figure 3 shows the microwave reflectance $R_{s}$, transmittance $T_{\mathrm{s}}$, and absorption $A_{\mathrm{s}}(8)$ at $10 \mathrm{GHz}$ plotted directly as a function of $\sigma_{\mathrm{s}}$. This approach removes ambiguity associated



Figure 3. Microwave transmittance $T_{s}$ (triangles), reflectance $R_{s}$ (solid squares) and absorption $A_{s}$ (circles) of Au films at $10 \mathrm{GHz}$ in relation to the film surface conductance $\sigma_{s}$. 
with correlating the measured mass film thickness with surface coverage in semicontinuous films. Reflectance of films with conductance $\sigma_{\mathrm{s}}<3 \times 10^{-4} \mathrm{~S}$ is near zero. In this dielectric regime, the transmittance gradually decreases from $100 \%$ to about $70 \%$, which is balanced by a gradual increase in absorption, $A_{\mathrm{s}}$. Within percolation transition when $\sigma_{\mathrm{s}}$ increases from $3 \times 10^{-4} \mathrm{~S}$ to $10^{-2} \mathrm{~S}$, the transmittance falls rapidly to zero and $A_{\mathrm{s}}$ shows a peak reaching a maximum of about 0.5 at $\sigma_{\mathrm{s}} \approx$ $3 \times 10^{-3} \mathrm{~S}$, while $\mathrm{R}_{\mathrm{s}}$ increases steeply. Above the conductivity percolation transition, when $\sigma_{\mathrm{s}}>10^{-2} \mathrm{~S}, A_{\mathrm{s}}$ decays reaching a value of about $10 \%$ at $\sigma_{\mathrm{s}} \approx 0.1 \mathrm{~S}$, while $\mathrm{R}_{\mathrm{s}}$ approaches a value of $90 \%$. The large value of reflectance above $\sigma_{\mathrm{s}} \approx 0.01 \mathrm{~S}(d>$ $10 \mathrm{~nm}$ ) is consistent with the film forming an electrically conducting mesh. The size of the conducting clusters, estimated from our microscopic study is about $25 \mathrm{~nm}$ [13]. Thus, the metallic mesh at wavelengths $\lambda$ longer than the cluster size is almost totally reflecting. In Figure $3, R_{\mathrm{s}}>90 \%$ when $\sigma_{\mathrm{s}} \approx 0.15$ $\mathrm{S}(d>12 \mathrm{~nm})$ and the network of $\mathrm{Au}$ nanoparticles acquires electrical characteristics of a continuous metallic film, with the bulk conductivity $\sigma_{\mathrm{v}} \approx 10^{7} \mathrm{~S} / \mathrm{m}$.

\section{DISCUSSION}

The absorption peak that appears within the conductivity percolation transition (Figure 3) is an interesting characteristic of the semicontinuous randomly distributed conductive nanoparticles. To our knowledge this is the first experimental evidence of such absorption in the microwave range. The results indicate a non-classical effect where microwave transmittance falls far more rapidly than the classical skin depth model [18] would suggest. Table 2 illustrates this situation where attenuation $1-T_{\delta \mathrm{e}}=1-\exp \left(-2 d / \delta_{\mathrm{e}}\right)$, due to skin penetration depth, $\delta_{\mathrm{e}}=\left(\pi f \mu_{\mathrm{r}} \mu_{0}\left(\sigma_{\mathrm{s}} / d\right)\right)^{-1 / 2}$, is several orders of magnitude smaller than the actually measured absorption $A_{\mathrm{s}}$.

For $8 \mathrm{~nm}$ thick films, the classical attenuation model predicts absorption of $2 \times 10^{-3}$, since at $10 \mathrm{GHz} \sigma_{\mathrm{s}} \approx 3 \times 10^{-3} \mathrm{~S}$ and the skin depth $\delta_{\mathrm{e}}$ is about $9 \mu \mathrm{m}$. In comparison, the measured absorption approaches a value of 0.5 and the corresponding classical penetration depth $\delta_{\mathrm{As}}=-2 d / \ln \left(1-A_{\mathrm{s}}\right)$ decreases to only about $25 \mathrm{~nm}$.

Large local electromagnetic field fluctuations have been identified numerically at the percolation transition in $2 \mathrm{D}$ metallic-dielectric networks, assuming a strong skin effect, and frequency independent conductivity [2]. The presented microwave scattering data provide experimental evidence that the interaction of the electromagnetic field with randomly distributed conductive particles leads to localized resonant modes within the film, confined in a dimension comparable

Table 2. Surface conductance $\left(\sigma_{s}\right)$, skin depth $\left(\delta_{\mathrm{e}}\right)$, attenuation $\left(1-\mathrm{T}_{\delta_{\mathrm{e}}}\right)$, measured absorption $\left(A_{s}\right)$, and the corresponding penetration depth $\delta_{\mathrm{As}}$ at $10 \mathrm{GHz}$ for Au films having thickness (d).

\begin{tabular}{|c|c|c|c|c|c|}
\hline $\begin{array}{c}d \\
(\mathrm{~nm})\end{array}$ & $\begin{array}{c}\sigma_{\mathrm{s}} \\
(\mathrm{S})\end{array}$ & $\delta_{\mathrm{e}}(\mu \mathrm{m})$ & $1-T_{\delta_{\mathrm{e}}}$ & $A_{\mathrm{s}}$ & $\begin{array}{c}\delta_{\mathrm{As}} \\
(\mathrm{nm})\end{array}$ \\
\hline 7 & $1.5 \times 10^{-5}$ & 109 & $1.3 \times 10^{-4}$ & 0.05 & 190 \\
\hline 8 & $3 \times 10^{-3}$ & 9 & $2 \times 10^{-3}$ & 0.5 & 25 \\
\hline 9 & $1.5 \times 10^{-2}$ & 4 & $5 \times 10^{-3}$ & 0.35 & 40 \\
\hline 10 & $5 \times 10^{-2}$ & 2.3 & $9 \times 10^{-3}$ & 0.2 & 90 \\
\hline
\end{tabular}

with the particle grain size, which is in the range of $20 \mathrm{~nm}$ to $100 \mathrm{~nm}$ (Table 2).

In an attempt to explore the origin of the microwave absorption in more detail, we examined the phase characteristic of the reflected wave, $\boldsymbol{S}_{11}$ shown in Figure 2. The phase angle $(\varphi)$ of the scattering parameter $\boldsymbol{S}_{11}$ is negative, consistent with the predominantly dielectric character of films thinner than 6 $\mathrm{nm}$ below the percolation threshold. With film conductance increasing beyond the conductivity percolation threshold, $\sigma_{\mathrm{s}}>$ $10^{-3} \mathrm{~S},(d>6 \mathrm{~nm})$ the phase angle changes from negative to positive values by about $\pi$. This indicates a transition from a capacitive $\left(X_{\mathrm{C}}\right)$ to inductive reactance $\left(X_{\mathrm{L}}\right)$ evidencing an LC resonance, which leads to power dissipation over the randomly distributed conducting paths. The film response may be formally described as equivalent to a distribution of resonant LC circuits or electrically shorted lossy transmission lines. The wide range of conductivity values over which the anomalous absorption is observed (Figure 3) suggests high damping conditions and that the distribution of the effective guided wavelengths is rather broad. The ratio of the internal film resistance and impedance, $|R / Z|$ normalizes the resonant absorption such that if $Z=\mathrm{R}$ then $A_{\mathrm{s}}=1.0$ [19]. When $A_{\mathrm{s}}$ attains a peak value $\approx 0.5$, the film reactance is inductive $(Z=R$ $\left.+X_{\mathrm{L}}\right)$ and $\mathrm{R} /\left(\mathrm{R}+X_{\mathrm{L}}\right) \approx 0.5$. The result demonstrates that a portion of the injected microwave power is trapped in the magnetic field. The 2D semicontinuous films composed of metallic gold nanoparticles act within the percolation conductivity transition as a surface reactance with diminishing resistive part.

Trapped mode resonances are characteristic of $2 \mathrm{D}$ metasurfaces, which are periodic arrays of planar resonant circuits with dimensions smaller than the wavelength. Metasurfaces or metafilms can be considered as a subset of $3 \mathrm{D}$ metamaterials. The properties of classical thin film metamaterials have been traditionally characterized by effective permittivity and permeability with the effective medium theory. Since the bulk properties and thickness of metasurfaces cannot be uniquely defined, the surface susceptibilities are used instead, and the scattering from metasurfaces is characterized in terms of the Generalized Sheet Transition Conditions (GSTC)[20]. The theoretical studies predict trapped mode resonance having a Fano-type line shape on the reflection/transmission spectra [21], [22]. One of the attractive features offered by metasurfaces is the possibility of compact electromagnetic absorbers. Such devices will scatter in a narrow band, in the vicinity of the resonant frequency range [23]. In contrast to GSTC results, the scattering parameters (Figure 2) and conductance (Table 1) of semicontinuous films are evidently frequency independent in the investigated frequency range suggesting that the characteristic properties of such films can be described by the effective medium (GEM) model. The GEM universal exponents $t \approx s \approx 1.2$ are consistent with $2 \mathrm{D}$ percolated network. But, using our experimental data, $\sigma_{\mathrm{v}} \approx 10^{7}$ $\mathrm{S} / \mathrm{m}, \varepsilon_{\mathrm{d}} \approx 10$, and $1 /(t+s)=0.42$, GEM predicts the effective absorption in unrealistically narrow concentration range, $\left|p-p_{\mathrm{c}}\right|<\left|2 \pi f \varepsilon_{0} \varepsilon_{\mathrm{d}} /\left(4 \pi \sigma_{\mathrm{v}}\right)\right|^{1 /\left(t_{+} s\right)}=8.6 \quad 10^{-4}$. To solve these difficulties, a dynamic effective model was introduced and the effect of broadband scattering of microwaves from inhomogeneous surfaces near percolation coverage has been analysed numerically in terms of generalized Ohm's law (GOL) [22]. The GOL simulations support our observation of an anomalous broadband absorption corresponding to resonating 
structures at the limit of high damping and strong skin penetration.

In practice one can easily estimate scattering properties of 2D semicontinuous films using surface conductance and $A_{s}, T_{\mathrm{s}}$ and $R_{s}$ plots in Figure 3. For example, transmission $T_{\mathrm{s}}$ of a single layer with $\sigma_{\mathrm{s}} \approx 3 \times 10^{-3} \mathrm{~S}$ is about 0.5 and $\mathrm{R}_{\mathrm{s}} \approx 0.14$. Neglecting multiple reflections in an n-layer configuration, transmission will decrease as $\left(T_{\mathrm{s}}\right)^{\mathrm{n}}, R_{\mathrm{s}}$ will remain about the same and the total absorption will approach the value of $1-\left(T_{\mathrm{s}}\right)^{\mathrm{n}}$ - $R_{\mathrm{s}}$. For a nano-size structure composed of 10 layers, $\left(T_{\mathrm{s}}\right)^{\mathrm{n}} \approx$ $10^{-3}(-30 \mathrm{~dB})$ and absorption will increases to about 0.85 . In comparison, a classical absorber with $R_{s}$ in the range of 0.14 and total attenuation, 1- $\left(T_{\mathrm{s}}\right)^{\mathrm{n}}$, of 0.997 would have to be macroscopically thick.

Somewhat more accurate results may be obtained from numerical models discussed above. Nevertheless, the presented results demonstrate the practical usefulness and advantage of our experimental approach, which utilizes surface conductance as a constituent parameter in characterization of anomalous microwave scattering from $2 \mathrm{D}$ semicontinuous films.

\section{CONCLUSIONS}

The presented measurement technique minimizes the impedance mismatch and allows effective coupling of microwaves to nanostructured metallic films over a broad frequency range. The results provide experimental evidence of large resonant absorption in semicontinuous gold films within the conductivity percolation transition. This transition can be easily identified experimentally from the characteristic $\pm \pi$ phase change of the reflection scattering parameter $S_{11}$. The effective attenuation distance of microwaves is about $25 \mathrm{~nm}$ to $100 \mathrm{~nm}$. This is several orders of magnitude shorter than the classical skin depth model would predict. The films attain a maximum absorption of about 0.5 slightly above the percolation threshold, when the film thickness is about $8 \mathrm{~nm}$ and surface conductance is about $2.5 \times 10^{-3} \mathrm{~S}$. By tuning the particle concentration towards the percolation threshold, the film reactance becomes inductive and the structure transitions from an electric to magnetic energy concentrator. The effect opens the possibility of controlled scattering of microwave energy in sub-wavelength structures through networks of metallic nanoparticles. Conductance of films thicker than $15 \mathrm{~nm}$ approaches the value of $0.6 \mathrm{~S}$, which corresponds to volume conductivity of bulk metallic gold of about $10^{7} \mathrm{~S} / \mathrm{m}$.

\section{DISCLAIMER}

Certain commercial equipment, instruments, or materials are identified in this paper to foster understanding. Such identification does not imply recommendation or endorsement by the National Institute of Standards and Technology, nor does it imply that the materials or equipment identified are necessarily the best available for the purpose.

\section{REFERENCES}

[1] V.M. Shalaev, "Nonlinear Optics of Random Media: Fractal Composites and Metal-Dielectric Films", Springer-Verlag Berlin.

[2] V.A. Shubin, A.K. Sarychev, J.P. Clerc, V.M. Shalev, "Local Electric and Magnetic Fields in Semicontinuous Metal Films: Beyond the Quasistatic Approximation", Phys. Rev. B 62 (2000) pp. 11230-11244.
[3] N. Kaiser, "Review of Fundamentals of Thin Film Growth", Appl. Optics 41 (2002) pp 3053-3059.

[4] M. Hovel, B. Gompf, M. Dressel, "Dielectric Properties of Metal Films Around the Percolation Threshold, Phys. Rev. B 81 (2010) p. 035402.

[5] V. Krachmalnicoff, E. Castanie, Y. De. Widde, R. Carminati, "Fluctuations of the Local Density of States Probe Localized Surface Plasmons in Disordered Metal Films" Phys. Rev. Lett. 105 (2010) p. 183901.

[6] M. Walther, D. G. Cooke, C. Sherstan, M. Hajar, M. R. Freeman, F. A. Hegmann, "Terahertz Conductivity of Thin Gold Films at the Metal-Insulator Percolation Transition" Phys. Rev. B 76 (2007) p. 125408.

[7] A. N. Lagarkov, K.N. Rozanov, A.K. Sarychev, N.A. Simonov, "Experimental and Theoretical Study of Metal-Dielectric Percolating Films at Microwaves" Physica A 241 (1997) pp. 199-206.

[8] I. R. Hooper, J.R. Sambles, "Some Considerations on the Transmissivity of Thin Metal Films" Opt. Express 16 (2008) pp.17249-17257.

[9] J. Obrzut, O. Kirillov, "Microwave Conductance of Semicontinuous Metallic Films from Coplanar Waveguide Scattering Parameters" 2013 IEEE International Instrumentation and Measurement Technology Conference Proceedings, Minneapolis, MN, May 6-9, 2013, pp. 912-916.

[10] J. Obrzut, "General Analysis of Microwave Network Scattering Parameters for Characterization of Thin Film Materials" Measurement J., 46 (2013) pp. 2963-2970.

[11] A. M. Nicolson, "Measurement of the intrinsic properties of materials by time-domain technique" IEEE Trans. Instr. Meas. 19 (1970) pp. 377-382.

[12] W. R. Eisenstadt, Y. Eo, S-Parameter-Based IC Interconnect Transmission Line Characterization, IEEE Trans. Compon, Hybrids Manuf. Technol. 15 (1992) pp. 483-490.

[13] J. Obrzut, J. F. Douglas, O. Kirilov, F. Sharifi, J. A. Liddle," Resonant Microwave Absorption in Thermally Deposited Au Nanoparticle Films Near Percolation Coverage", Langmuir, 29, (2013) pp. 9010-9015.

[14] S. Galam, A. Mauger, "Universal Formulas for Percolation Threshold”, Phys. Rev. E 53 (1996) pp. 2177-2181.

[15] D. M. Grannan, J.C. Garland, D.B. Tanner, "Critical Behaviour of the Dielectric Constant of a Random Composite near the Percolation Threshold" Phys. Rev. Lett. 46 (1981) pp. 375 -378.

[16] D. S. McLachlan, G. Sauti. C. Chiteme, "Static Dielectric Function and Scaling of the AC Conductivity for Universal and Nonuniversal Percolation Systems", Phys. Rev. B 76 (2007) p. 014201.

[17] D. Simien, J.A. Fagan, W. Luo, J.F. Douglas, K.M. Migler, J. Obrzut, "Influence of Nanotube Length on the Optical and Conductivity Properties of Thin Single-Wall Carbon Nanotube Networks", ACS NANO 2 (2008) pp. 1879-1884.

[18] J. D. Jackson, Classical Electrodynamics, Wiley, NY (1999), Section 5.18

[19] M. Sucher, "Measurement of Q", In Handbook of Microwave Measurements; Sucher, M.; and Fox, J., Eds.; Polytechnic Press of the Polytechnic Institute of Brooklyn: New York (1963) p. 420.

[20] C. L. Holloway, E. F. Kuester, J. G. Gordon, J. O'Hara, J. Booth and D. R. Smith, "An Overview of the Theory and Applications of Metasurfaces: The Two-Dimensional Equivalents of Metamaterials", IEEE Antenna and Prop. Mag., 54 (2012) pp. 10-34.

[21] V. A. Fedotov, M. Rose, S. L. Prosvirnin, N. Papasimakis and N. I. Zheludev, "Sharp Trapped Mode Resonances in Planar Metamaterials with a Broken Structural Symmetry", Phys. Rev. Lett., 99 (2007) p. 147401.

[22] A. Dhoubi, S. N. Burokur, A. Lupu, A. de Lustrac and A. Priou, "Excitation of Trapped Modes from a Metasurface Composed of only Z-shaped Meta-Atoms", Appl. Phys. Lett., 103 (2013) p. 184103.

[23] O. Luukkonen, F. Costa, C. R. Smovski, A. Monorchio and S. A. Tretyakov, "A Thin Electromagnetic Absorber for Wide Incidence Angles and Both Polarizations", IEEE Trans. Antennas Propag., 57 (2009) pp. 3119-3125. 